\title{
Power Prediction and Technoeconomic Analysis of a Solar PV Power Plant by MLP-ABC and COMFAR III, considering Cloudy Weather Conditions
}

\author{
M. Khademi, ${ }^{1}$ M. Moadel, ${ }^{2}$ and A. Khosravi $^{3}$ \\ ${ }^{1}$ Department of Applied Mathematics, Islamic Azad University, South Tehran Branch, No. 209, North Iranshahr Street, \\ Tehran 11365-4435, Iran \\ ${ }^{2}$ Department of Energy Systems Engineering, Islamic Azad University, South Tehran Branch, No. 209, North Iranshahr Street, \\ Tehran 11365-4435, Iran \\ ${ }^{3}$ Islamic Azad University, South Tehran Branch, No. 209, North Iranshahr Street, Tehran 11365-4435, Iran \\ Correspondence should be addressed to M. Khademi; khademi@azad.ac.ir
}

Received 4 December 2015; Revised 24 January 2016; Accepted 31 January 2016

Academic Editor: Pouria Ahmadi

Copyright @ 2016 M. Khademi et al. This is an open access article distributed under the Creative Commons Attribution License, which permits unrestricted use, distribution, and reproduction in any medium, provided the original work is properly cited.

\begin{abstract}
The prediction of power generated by photovoltaic (PV) panels in different climates is of great importance. The aim of this paper is to predict the output power of a $3.2 \mathrm{~kW}$ PV power plant using the MLP-ABC (multilayer perceptron-artificial bee colony) algorithm. Experimental data (ambient temperature, solar radiation, and relative humidity) was gathered at five-minute intervals from Tehran University's PV Power Plant from September 22nd, 2012, to January 14th, 2013. Following data validation, 10665 data sets, equivalent to 35 days, were used in the analysis. The output power was predicted using the MLP-ABC algorithm with the mean absolute percentage error (MAPE), the mean bias error (MBE), and correlation coefficient $\left(R^{2}\right)$, of 3.7, 3.1, and $94.7 \%$, respectively. The optimized configuration of the network consisted of two hidden layers. The first layer had four neurons and the second had two neurons. A detailed economic analysis is also presented for sunny and cloudy weather conditions using COMFAR III software. A detailed cost analysis indicated that the total investment's payback period would be 3.83 years in sunny periods and 4.08 years in cloudy periods. The results showed that the solar PV power plant is feasible from an economic point of view in both cloudy and sunny weather conditions.
\end{abstract}

\section{Introduction}

Photovoltaic cells collect sunlight and convert it to electrical energy, which is the most convenient way to utilize solar energy. The performance of a PV panel is strongly dependent on the availability of solar irradiance at the required location, PV panel temperature, and other environmental conditions. Thus, reliable knowledge and understanding of the PV panels' performance under different operating conditions are of great importance for accurate prediction of their energy output and correct site selection [1].

In recent years, large numbers of research projects have been carried out relating to the prediction of a solar PV system's efficiency and optimizing the effective parameters with the use of artificial intelligence techniques [2]. Some studies have been done in order to investigate the environmental factors which affect the current-voltage $(I-V)$ characteristics of PV modules based on the simultaneous measurement of the open-circuit voltage $V_{\mathrm{oc}}$ as a function of a slowly varying light intensity [3]. Bayrakci et al. [4] analyzed the effect of temperature on the performance of PV modules. There are also some power efficiency models [5] that can predict the real dynamic or average performance of a PV system under variable climatic conditions [6].

The main disadvantage of mathematical modeling is the dependency of the model's parameters on operating conditions; that is, a given set of operating conditions needs a corresponding set of parameters. This weak point largely limits the application of the model. Additionally, there is no specific mathematical model for the prediction of $\mathrm{PV}$ 

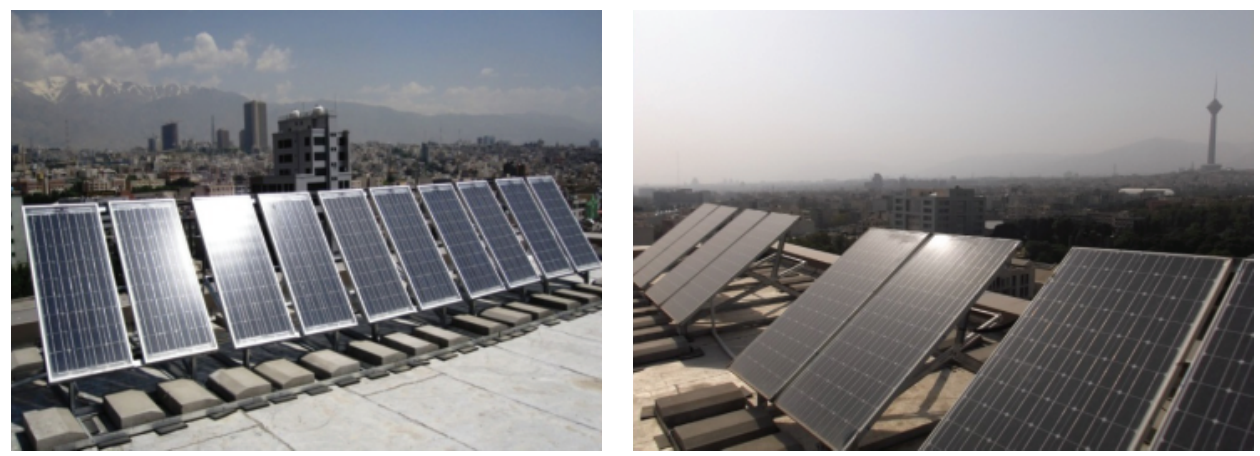

FIgure 1: University of Tehran's Photovoltaic Plant Complex (Source: http://pvlab.ut.ac.ir).

output in cloudy conditions. Many artificial neural networks (ANNs), which use only one algorithm for different weather conditions, have been developed in order to find the optimum operating point of PV panels [7-9]. The research that has been carried out in the field of ANNs shows that with more accurate data classification and the development of a special algorithm for each classification, better results can be achieved.

By increasing the forecast accuracy of output power of the solar panels, better control could be achieved with greater precision. This would allow us to increase the energy efficiency of photovoltaic panels and make them more cost effective $[10,11]$. The results could be used in order to build an intelligent controller, which could find the maximum power point (MPP) [12-14]. The controller would find the MPP according to the ANN prediction when the radiation decreases caused by sudden cloud coverage. The regions with wet climatic conditions with cloudy skies, such as the Mediterranean or the north of Iran, are suitable for study by this approach in order to optimize the output energy prediction of the panels.

The main objective of this study was to pursue a simplified simulation model, with acceptable levels of precision, in order to predict the output power of PV modules under different operating conditions, giving particular consideration to the sudden occurrence of clouds. For this purpose, the classification method was applied and data was divided into two groups: cloudy data and sunny data. The financial indexes of a 20-year life cycle of the PV power plant, considering sunny days and cloudy periods, were calculated and compared. In this paper, MLP-ABC algorithms were used to predict the output energy of solar panels. The obtained results show that these methods can be used instead of time-consuming experimental tests to determine the PV panels' output energy with a desirable level of accuracy. A detailed economic analysis was also carried out using COMFAR III software.

\section{Material and Method}

2.1. Data. Data was measured and registered by data loggers at five-minute intervals and was taken from the Tehran University Photovoltaic Power Plant, located in Tehran, Iran, at a longitude of $\mathrm{N}^{\circ} 37.51$, latitude of $\mathrm{E}^{\circ} 47.35$, and an altitude of 1548 meters (see Figure 1).

In this study, ambient temperature, relative humidity, incoming radiation, and PV output power between September 22nd, 2012, and January 14th, 2013, was used. The validation test was done on the data to verify the accuracy of the data's registration. To accomplish this, incoming radiations were compared with extraterrestrial radiation. The measured power was integrated to calculate the total obtained energy within each day and the nominal power values of PV modules were then compared to one another.

\section{MLP (Multilayer Perceptron) Neural Network}

An MLP neural network was used for data classification. MLPs are composed of input layers, hidden layers, and output layers, which contain certain neurons (see Figure 2). This MLP build, to calculate the power of the solar array, had an output layer comprising of two neurons for calculating the MPP that represent the output voltage and current, corresponding to the maximum power point of the array. We used 215 samples for training and 104 samples for testing the network. In the MLP neural network training process for data classification categories, the ultimate goal is to find the best neural network weights that could be terminated to the smallest Mean Square Error (MSE). In this paper, the reduced gradient method was proposed for finding the optimal weights for the neural network. The outputs corresponding to these inputs are compared with outputs from the network. If the difference between these two values is lesser, the network will be better trained.

\section{MLP-ABC (Multilayer Perceptron Neural Network with ABC Algorithm)}

The bee colony algorithm was proposed in 2005 by Karaboga. This algorithm was inspired by the exploratory behavior of bee colonies, which is similar to other intelligent group methods. It uses a collection of certain individuals within a group that alone have not been specifically intelligent. 


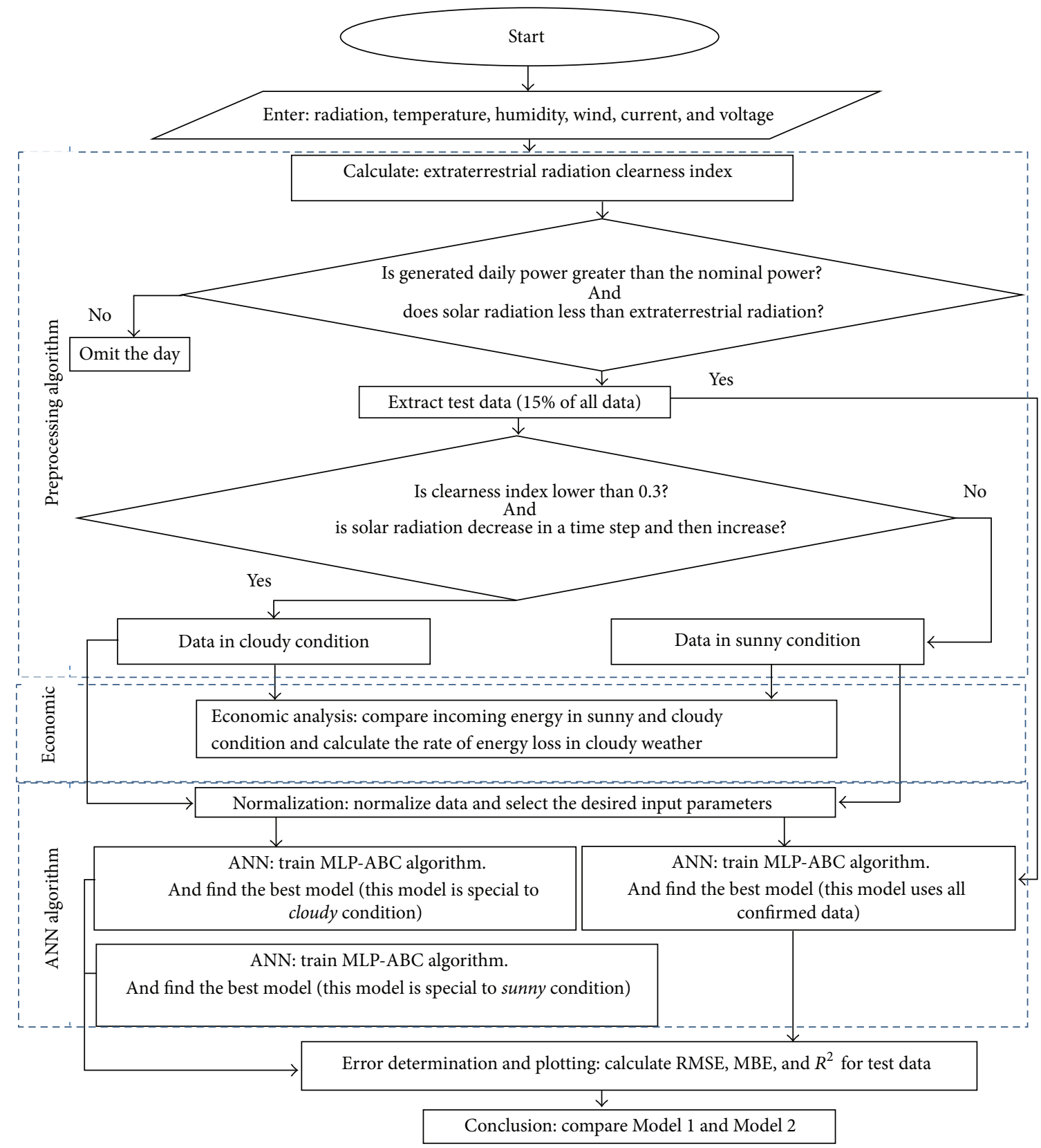

FIGURE 2: Data selection method, preparation for feed-forward neural network, and output result.

Both employed and scot bees work together to find the best solution to the problems. The first one works on the current solution and those around it hoping for an improved result. In the case of no acceptable result, an employed bee becomes a scot and tries to look for another solution in areas farther away. The ABC algorithm is used to solve continuous optimization problems and find the optimum of a function or a combination of multiple scalar functions [15]. The main steps of the $\mathrm{ABC}$ algorithm are used as per Aryan et al. [16].

MLP finds its best weights via a gradient descent approach, in which they are computed by backpropagation of errors (different between the network outputs and the desired ones) through the network. However, since it is a problem of optimization, $\mathrm{ABC}$ can be utilized instead of the backpropagation approach. In MLP-ABC, weights are computed by collection of bee agents. They look for the optimum combination of weights in the solution space that results in the best network for the problem.

In order to apply the MLP-ABC model, input parameters are air temperature $\left({ }^{\circ} \mathrm{C}\right)$, irradiance $\left(\mathrm{W} / \mathrm{m}^{2}\right)$, and relative humidity (\%) and the output is power. The model consists of two hidden layers. The first layer has four neurons and the second has two neurons.

4.1. The Underlying Assumption and Data Selection. Determining the cloudiness of the sky requires complex and costly 
TABLE 1: The statistical classification of cloud database.

\begin{tabular}{lcccc}
\hline Criteria & Power $(\mathrm{W})$ & $\begin{array}{c}\text { Solar radiation } \\
\left(\mathrm{W} / \mathrm{m}^{2}\right)\end{array}$ & $\begin{array}{c}\text { Ambient temperature } \\
\left({ }^{\circ} \mathrm{C}\right)\end{array}$ & Relative humidity $(\%)$ \\
\hline Average & 841 & 99.8 & 17.05 & 44.73 \\
Maximum & 2724 & 499.7 & 30.40 & 99.9 \\
Minimum & 0 & 5.03 & -5.1 & 21.35 \\
Standard deviation & 718.7 & 86.3 & 7.83 & 18.16 \\
\hline
\end{tabular}

devices. However, as it is well known, the incoming solar radiation is directly related to the level of cloudiness. The clearness index $\left(K_{t}\right)$ is the criteria (amount of the total solar radiation on a horizontal surface at the surface of the Earth, divided by the corresponding irradiance available outside of the atmosphere) for determining the sudden cloud appearances. The key point is that the clear index changes during a sunny day. For instance, the color index is 0.25 at the 7:00 solar time, but it becomes 0.65 at the 12:00 solar time. The average of $K_{t}$ was therefore calculated for different times in a day. It is assumed that in any given moment, whenever $K_{t}$ becomes $40 \%$ lower than the long-term average of $K_{t}$, it is considered that cloud coverage is occurring.

As a more precise criterion, if, in a short time period (less than 5 minutes), the irradiance decreases and then increases, this time period is supposed to be the shade effect on the photovoltaic panel. This assumption may lead to errors in determining the correct time of sudden clouds. Dust, fleeting shadows, and other factors could also cause such situations. To minimize this error, both of the above criteria were used simultaneously. This means that whenever $K_{t}$ decreases while the amount of radiation fluctuates, the conditions are considered to be cloudy. With regard to this fact, 3,090 sets of data demonstrated cloudy conditions. However, since the aim was to predict the effect of cloudy conditions on decreasing irradiance and output of the panel, the assumption is acceptable.

Table 1 indicates the statistical classification of cloud databases. Daily data analysis indicates that in days of clear sky with neither cloud nor dust, solar radiation at 12:00 (which is considered as the maximum irradiance that could be achieved) varies between $850 \mathrm{~W}$ and $1100 \mathrm{~W}$. The data during cloudy days shows the maximum irradiance as $499.7 \mathrm{~W}$, which indicates the impact of clouds on the incidental radiation. Consequently, when the irradiance is associated with abnormal fluctuation, it could be considered the result of cloud occurrence over the panel. The idea of this research is not only to forecast the output power and energy of PV panels, but also to build a control system for these conditions. It could be a great help to optimize output power of PV panels when sudden cloud coverage occurs.

\section{Data Preparation}

The data was normalized between 0 and 1 for better network learning by the following equation:

$$
F(x)=\frac{X-X_{\min }}{X_{\max }-X_{\min }},
$$

TABLE 2: Comparison of results between two conditions (COMFAR's report).

\begin{tabular}{lcc}
\hline Economic index & Model \#1 & Model \#2 \\
\hline Total fixed investment (\$) & 150000000 & 150000000 \\
Net present value (NPV) (\$) & 10641,71 & 9914,05 \\
(discounting rate: 4\%) & $35,13 \%$ & $32,25 \%$ \\
Internal rate of return (IRR) & $17.40 \%$ & $18.29 \%$ \\
Break-even ratio (\%) & 3.83 years & 4.08 years \\
Normal payback period & 4.07 years & 4.36 years \\
Dynamic payback period & & \\
\hline
\end{tabular}

where $F(x)$ indicates the normalized value and $x$ indicates the actual value. The data was gathered over approximately four months. However, after applying data preprocessing, only 6,895 collections of "sunny" data and 3,090 collections of "cloudy" data remained for creating the MLP-ABC and 680 pieces of data for the testing of the networks. The mean absolute percentage error (MAPE) was used to compare the models. It is a measure of the accuracy of a method for constructing fitted time series values in statistics, specifically in trend estimation [17]. It usually expresses accuracy as a percentage which is defined as follows:

$$
\text { MAPE }=\frac{1}{N} \sum_{i=1}^{N} \frac{\left(X_{i-x_{i}}\right)}{x_{i}} .
$$

Mean bias error (MBE) and correlation coefficient $\left(R^{2}\right)$ were calculated by the following:

$$
\begin{aligned}
\mathrm{MBE} & =\sum_{i=1}^{N} \frac{\left(X_{i-x_{i}}\right)}{x_{i}}, \\
R^{2} & =\frac{\sum_{i=1}^{N}\left(x_{i}-\bar{X}\right)\left(X_{i}-\bar{X}\right)}{\left(\sqrt{\sum_{i=1}^{N}\left(x_{i}-\bar{X}\right)}\right) \times\left(\sqrt{\sum_{i=1}^{N}\left(X_{i}-\bar{X}\right)}\right)} .
\end{aligned}
$$

\section{Economic Analysis}

In this case study, the lifetime economic analyses of PV panels using MLP-ABC algorithms for sunny and cloudy periods were compared.

Net present value (NPV), internal rate of return (IRR), payback period $(\mathrm{PBP})$, and the balance sheet of the project were prepared according to accepted standards based on a 20year useful life. The details can be found in the full version of COMFAR's report; however, a brief summary is presented in Table 2. 


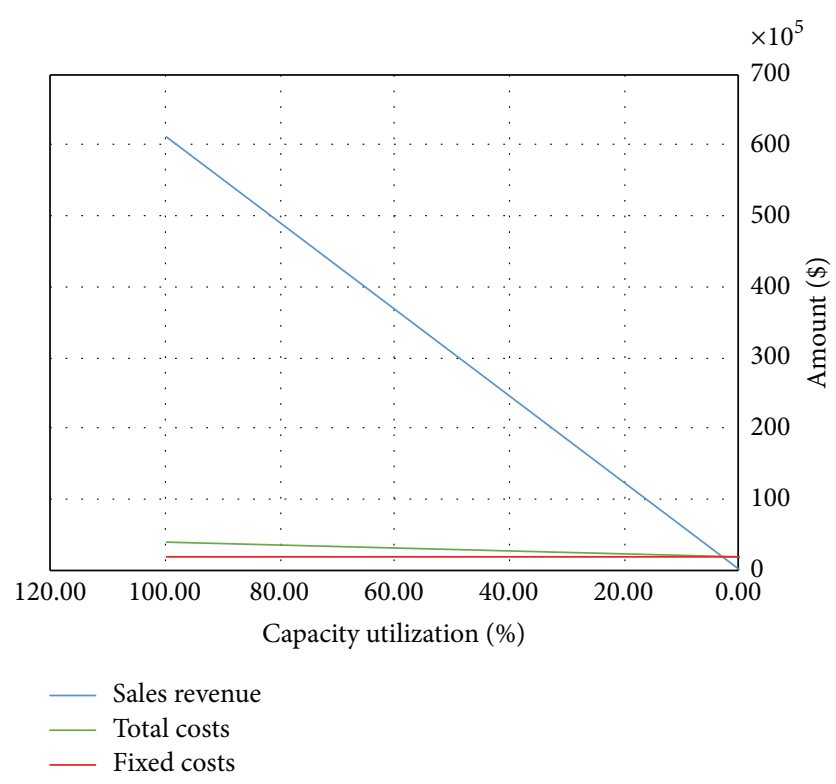

FIgURE 3: Break-even analysis for Model \#1.

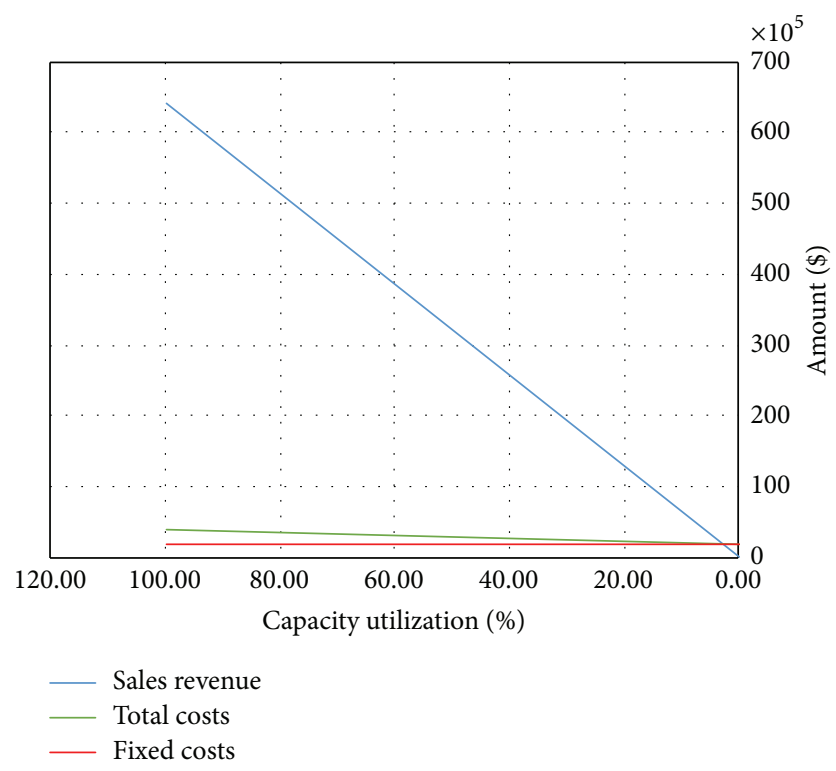

Figure 4: Break-even analysis for Model \#2.

A useful tool in tracking the cash flow of a business is a break-even analysis. This point is important to determine the price of power in order for the PV to still generate a contribution. The break-even analysis of the investment is performed to determine how many years it takes to generate enough contribution to cover the fixed and variable costs [18]. As the goal is to lower the break-even point and generate profit, it is important to understand this concept. Figures 3 and 4 show the break-even analysis for cloudy periods and sunny days, respectively. In this study, the best efficiency points (BEP) for sunny and cloudy periods are $17.40 \%$ and $18.29 \%$, respectively.

The internal rate of return, or discounted cash flow rate of return, offers analysts a way to quantify the rate of return provided by an investment. The COMFAR report shows that
TABLE 3: The generated error in PV panels' power prediction using MLP-ABC.

\begin{tabular}{lcccc}
\hline Model number & Model & MAPE (\%) & $R^{2}(\%)$ & MBE (W) \\
\hline 1 & $\begin{array}{c}\text { Sunny and } \\
\text { cloudy } \\
\text { (separately) }\end{array}$ & 3.7 & 94.7 & 3.1 \\
2 & $\begin{array}{c}\text { All conditions } \\
\text { together }\end{array}$ & 4.7 & 83.1 & 9.5 \\
\hline
\end{tabular}

the project is sufficiently feasible as it generates an IRR of $35.13 \%$ for sunny days. This is considered an attractive rate of return. The project IRR remains attractive even for the cloudy days as $32.25 \%$. Ultimately, IRR gives an investor the means to compare alternative investments based on their yield.

The panels' output power under cloudy conditions was measured and recorded. The effective clouds on the panel were divided into two categories: (1) clouds that cover the sky and have an effect on the panel for more than 2 hours and (2) parts of clouds that shade the panels for only a moment or several minutes (see Figure 5).

\section{Results and Discussion}

This study proposes an analysis to exhibit what happens when clouds pass over a solar power plant. The economic analysis, which is presented throughout this study, demonstrates the economic losses caused by sudden cloud coverage and shadows over the panel.

In this research, the effect of cloudy sky conditions on the energy produced by the panels is investigated. The energy generated by the panels during cloudy and sunny climatic conditions was separately predicted by artificial neural methods (MLP-ABC).

Two separate models were developed in different ways for the cloudy and sunny weather conditions. Error calculation and the evaluation of results showed that the first model for different conditions could significantly reduce errors (Table 3).

As mentioned, a more accurate output energy prediction of PV panels could increase the precision of energy supply planning and the design accuracy of control systems. Figures $6,7,8$, and 9 show the output power prediction of PV panels using the referred methods.

Figures 6 and 8 show the comparison between the predicted power by Model 1 and the measured power. As shown, this model also tracks the fluctuations of the clouds. Looking on a precise level, small fluctuations have occurred in the measured data where the power gets close to its maximum level. The developed model faces some difficulties in tracking these fluctuations. However, these fluctuations are not caused by cloud or external factors, and their value is very low. Thus, it is reasonable to ignore it.

Comparisons between Figures 7 and 9 show the effect of privatization data in order to reduce errors for sunny and cloudy conditions. The method that was demonstrated in the economic analysis section was used to determine the energy loss. Data was collected from September 22nd, 2012, to January 14th, 2013. 


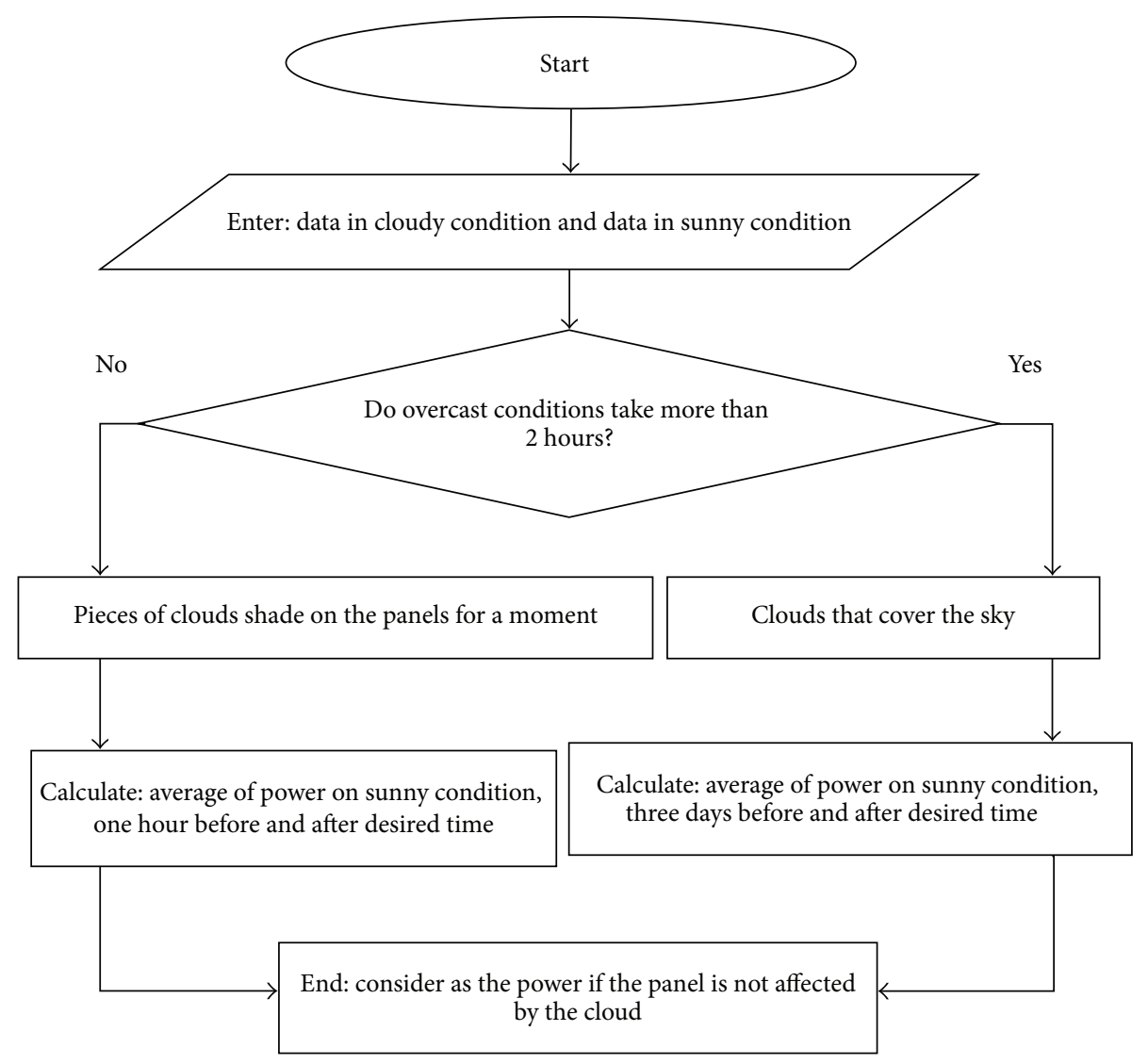

FIGURE 5: The method of determining the amount of power generated if the panel is not affected by clouds.

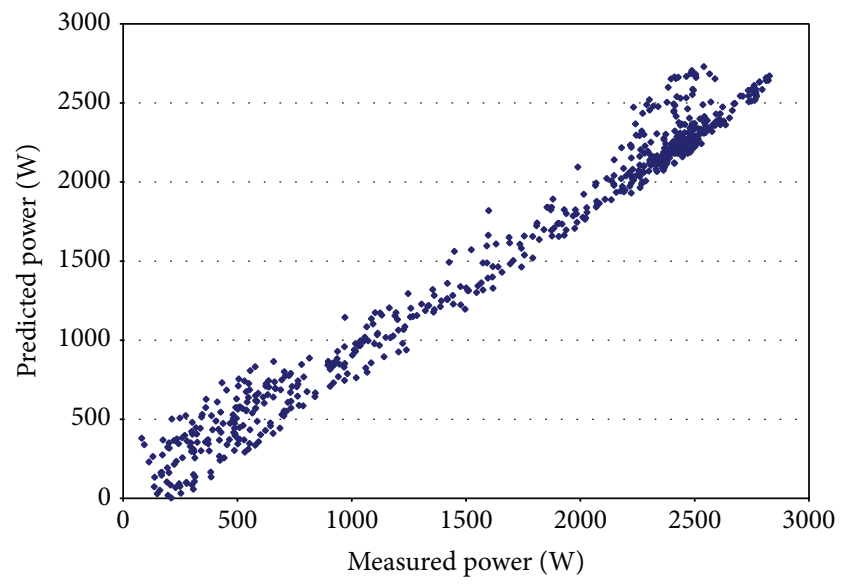

FIGURE 6: Output power prediction for Model \#1, which selects sunny and cloudy data randomly.

\section{Conclusions}

In this paper, the output energy of a $3.2 \mathrm{~kW} \mathrm{PV}$ solar power plant was predicted using an MLP-ABC algorithm and the results were compared with the experimental data. The ambient temperature, irradiance on the horizontal surface, and PV power (by multiplying current and voltage) were collected in the photovoltaic laboratory of Tehran University between September 22nd, 2012, and January 14th, 2013. 10,665

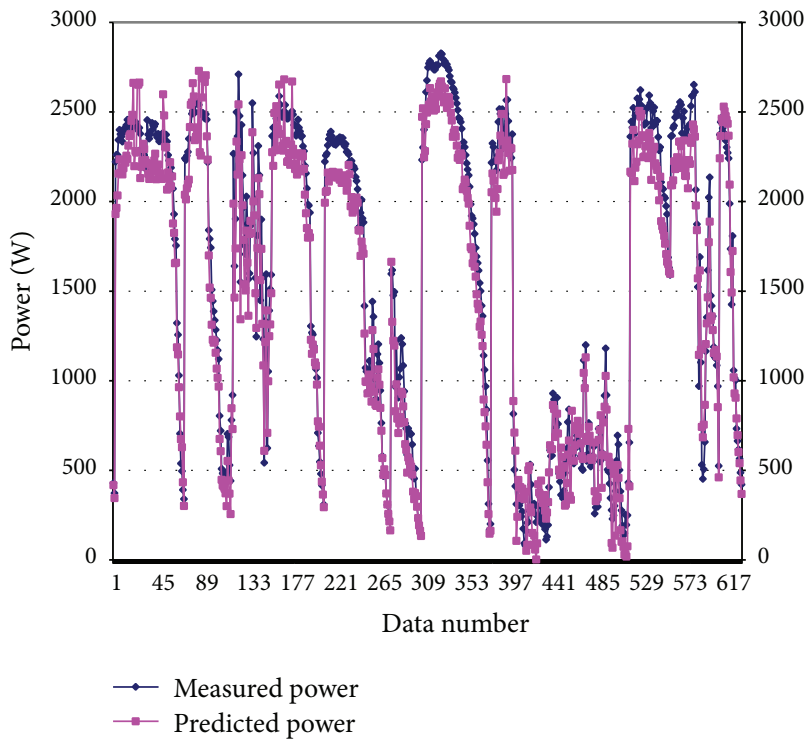

FIGURE 7: Output power prediction for Model \#1, which selects sunny and cloudy data randomly.

pieces of data were measured at five-minute intervals (over approximately 35 days) after preprocessing.

To improve the performance of the neural network, the sunny days were separated from the cloudy days. For clearness index values less than 0.3 , the day was considered as 


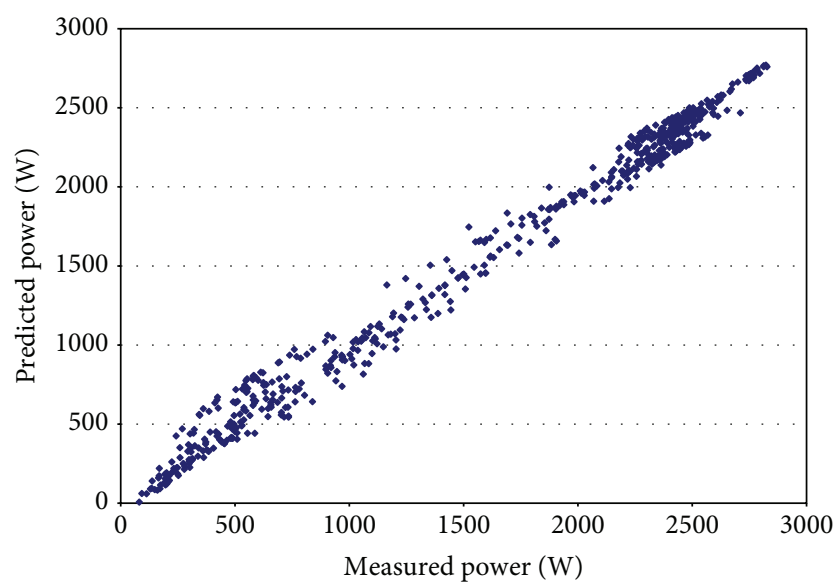

Figure 8: Output power prediction for Model \#2, which selects sunny and cloudy data separately.

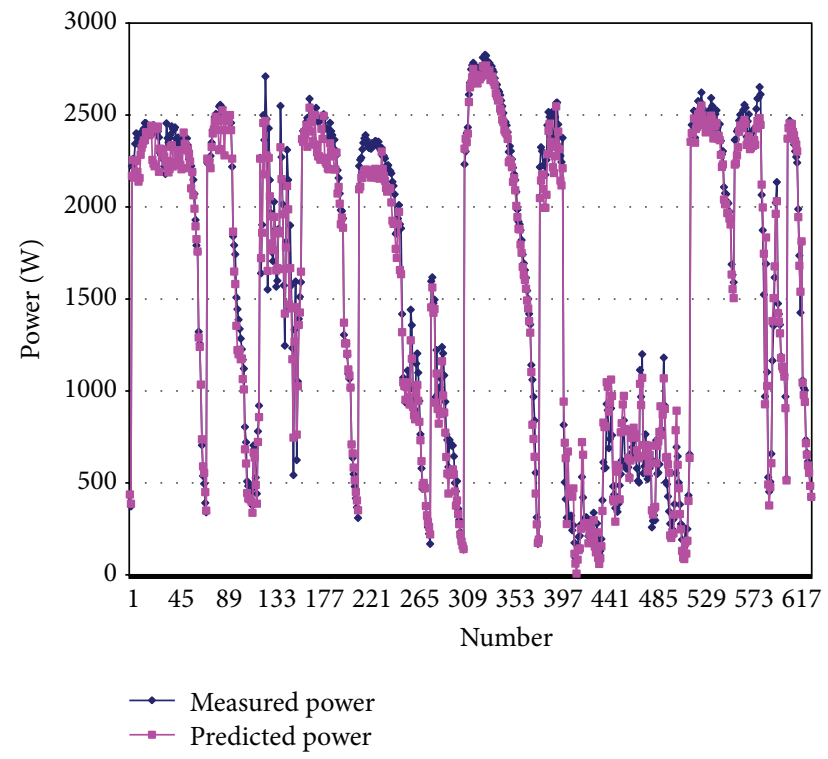

Figure 9: Output power prediction for Model \#2, which selects sunny and cloudy data separately.

sunny and if radiation fluctuation over a short time interval occurred, it was considered as cloudy time.

The output energy was predicted by two methods. In the first method, all sunny and cloudy data were used and acceptable results were obtained (MAPE $=4.7 \%, R^{2}=83.1 \%$, $\mathrm{MBE}=9.5$ ). In the second method, forecasting precision was improved significantly by separating the sunny and cloudy data $\left(\mathrm{MAPE}=3.7 \%, R^{2}=94.7 \%, \mathrm{MBE}=3.1\right)$.

The hypothesis was that neural network data classifications improve this model's performance. It has been concluded that with the weather forecast and the separation of different weather conditions, output power prediction can be done more accurately.

Within the period of this study, the solar power plant produced approximately $5237 \mathrm{kWh}$ /year of electricity in sunny weather conditions and $924 \mathrm{kWh} /$ year in cloudy weather conditions, with the energy loss due panel shading around $293 \mathrm{kWh} /$ year.
The results show that the project is feasible because of IRRs of $35.13 \%$ and $32.25 \%$ (for sunny and cloudy periods, resp.). These are considered to be attractive rates of return in capital markets. The investment has a payback period of 3.83 years in Model \#1 and 4.08 years in Model \#2.

\section{Conflict of Interests}

The authors declare that there is no conflict of interests regarding the publication of this paper.

\section{Acknowledgments}

This paper is based up on a research work supported by the Research Council of the Islamic Azad University South Tehran Branch (Contract no. 812). The authors are also grateful to Dr. Farzad Jafarkazemi for his academic advices and Alireza Nikookar and Ahmad Razeghi for their technical support.

\section{References}

[1] A. S. Joshi, I. Dincer, and B. V. Reddy, "Performance analysis of photovoltaic systems: a review," Renewable and Sustainable Energy Reviews, vol. 13, no. 8, pp. 1884-1897, 2009.

[2] M. M. Farzad Jafarkazemi, M. Khademi, and A. Razeghi, "Performance prediction of flat-plate solar collectors using MLP and ANFIS," Journal of Basic and Applied Scientific Research, vol. 3, no. 2, pp. 196-200, 2013.

[3] W. Zhou, H. Yang, and Z. Fang, "A novel model for photovoltaic array performance prediction," Applied Energy, vol. 84, no. 12, pp. 1187-1198, 2007.

[4] M. Bayrakci, Y. Choi, and J. R. S. Brownson, "Temperature dependent power modeling of photovoltaics," Energy Procedia, vol. 57, pp. 745-754, 2014.

[5] W. G. J. H. M. van Sark, "1.02-introduction to photovoltaic technology," in Comprehensive Renewable Energy, A. Sayigh, Ed., pp. 5-11, Elsevier, Oxford, UK, 2012.

[6] K. Ishaque and Z. Salam, "A review of maximum power point tracking techniques of PV system for uniform insolation and partial shading condition," Renewable and Sustainable Energy Reviews, vol. 19, pp. 475-488, 2013.

[7] A. Mellita and M. Benghanem, "Sizing of stand-alone photovoltaic systems using neural network adaptive model," Desalination, vol. 209, no. 1-3, pp. 64-72, 2007.

[8] A. Mellit and S. A. Kalogirou, "Artificial intelligence techniques for photovoltaic applications: a review," Progress in Energy and Combustion Science, vol. 34, no. 5, pp. 574-632, 2008.

[9] A. Mellit and S. A. Kalogirou, "MPPT-based artificial intelligence techniques for photovoltaic systems and its implementation into field programmable gate array chips: review of current status and future perspectives," Energy, vol. 70, pp. 1-21, 2014.

[10] H. Belmili, M. Haddadi, S. Bacha, M. F. Almi, and B. Bendib, "Sizing stand-alone photovoltaic-wind hybrid system: technoeconomic analysis and optimization," Renewable and Sustainable Energy Reviews, vol. 30, pp. 821-832, 2014.

[11] E. İzgi, A. Öztopal, B. Yerli, M. K. Kaymak, and A. D. Şahin, "Short-mid-term solar power prediction by using artificial neural networks," Solar Energy, vol. 86, no. 2, pp. 725-733, 2012. 
[12] J. Ma, K. L. Manb, T. O. Tingb, N. Zhangb, S.-U. Guanb, and P. W. H. Wonga, "DEM: direct estimation method for photovoltaic ximum power point tracking," Procedia Computer Science, vol. 17, pp. 537-544, 2013.

[13] P. Bhatnagar and R. K. Nema, "Maximum power point tracking control techniques: state-of-the-art in photovoltaic applications," Renewable and Sustainable Energy Reviews, vol. 23, pp. 224-241, 2013.

[14] L. L. Jiang, D. R. Nayanasiri, D. L. Maskell, and D. M. Vilathgamuwa, "A hybrid maximum power point tracking for partially shaded photovoltaic systems in the tropics," Renewable Energy, vol. 76, pp. 53-65, 2015.

[15] A. Abraham and B. Nath, "Hybrid heuristics for optimal design of artificial neural networks," in Developments in Soft Computing, vol. 9 of Advances in Soft Computing, pp. 15-22, Springer, Berlin, Germany, 2001.

[16] H. Aryan, M. Khademi, and M. Pedram, "Prediction of photovoltaic panels output power by using MLP, RNN and neuroevolution models," Advances in Natural and Applied Sciences, vol. 8, no. 14, pp. 74-81, 2014.

[17] D. T. Larose, Discovering Knowledge in Data, an Introduction to Data Mining, John Wiley \& Sons, 2005.

[18] O. Ekren, B. Y. Ekren, and B. Ozerdem, "Break-even analysis and size optimization of a PV/wind hybrid energy conversion system with battery storage-a case study," Applied Energy, vol. 86, no. 7-8, pp. 1043-1054, 2009. 


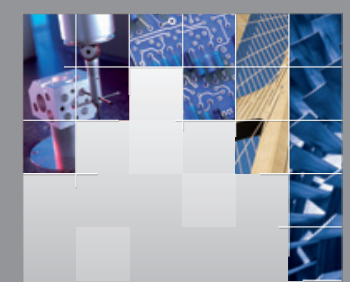

\section{Enfincering}
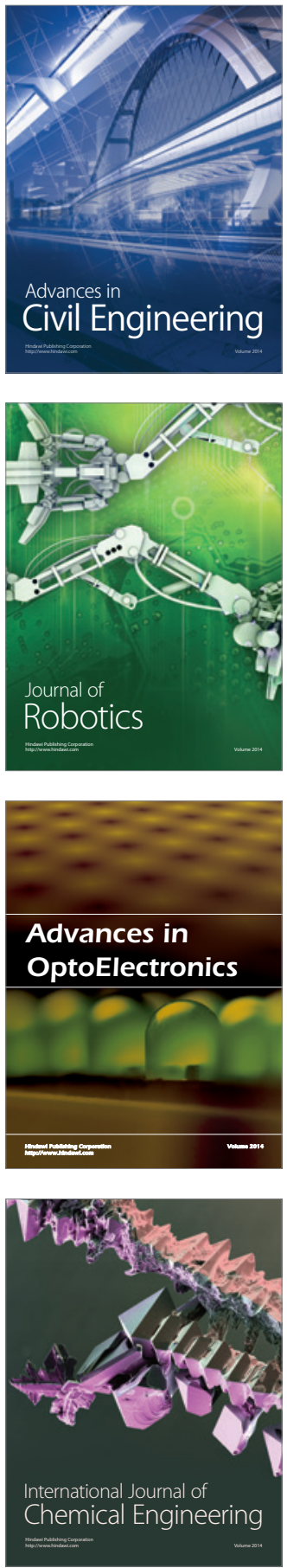

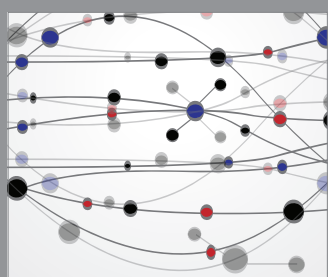

The Scientific World Journal

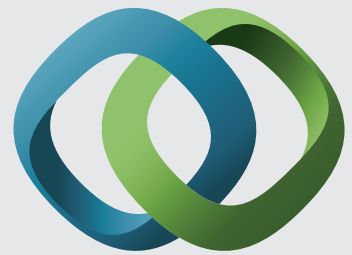

\section{Hindawi}

Submit your manuscripts at

http://www.hindawi.com
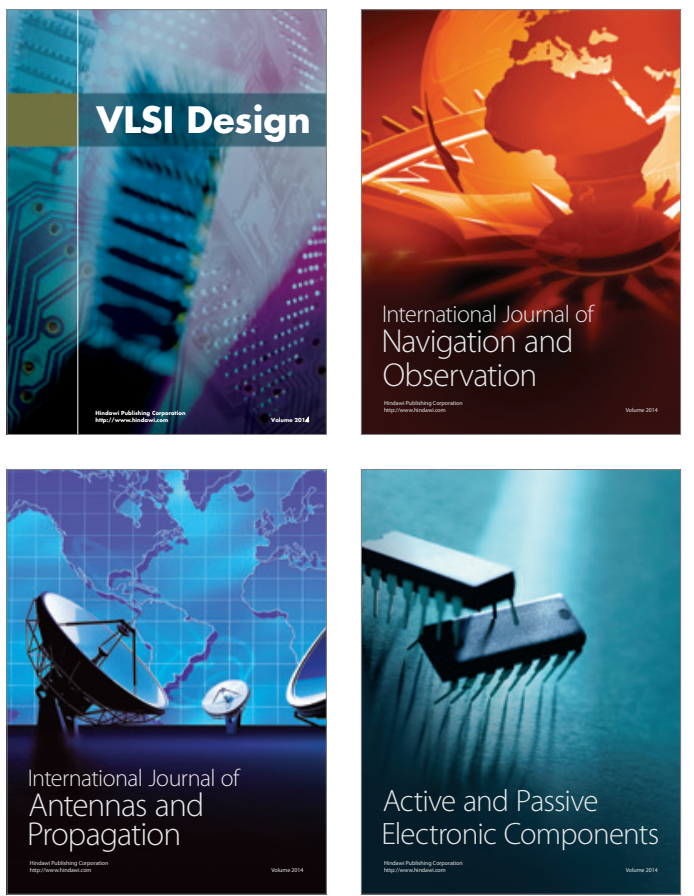
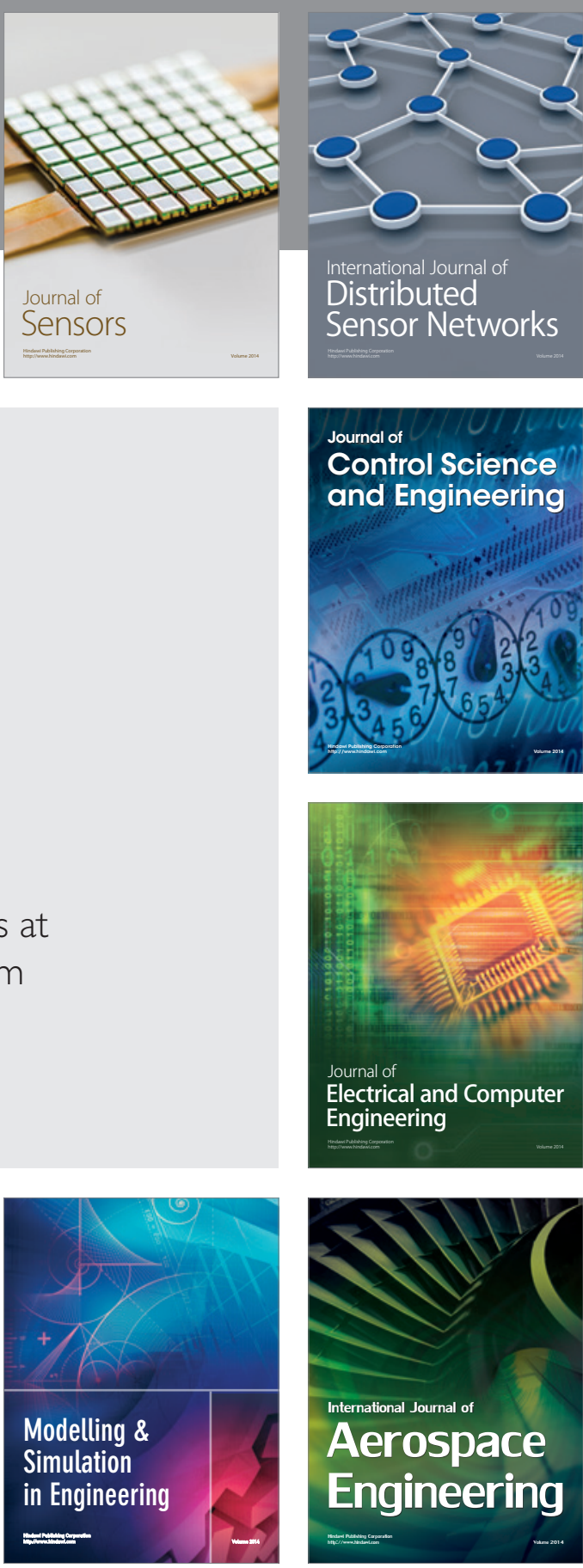

International Journal of

Distributed

Sensor Networks

Journal of

Control Science

and Engineering
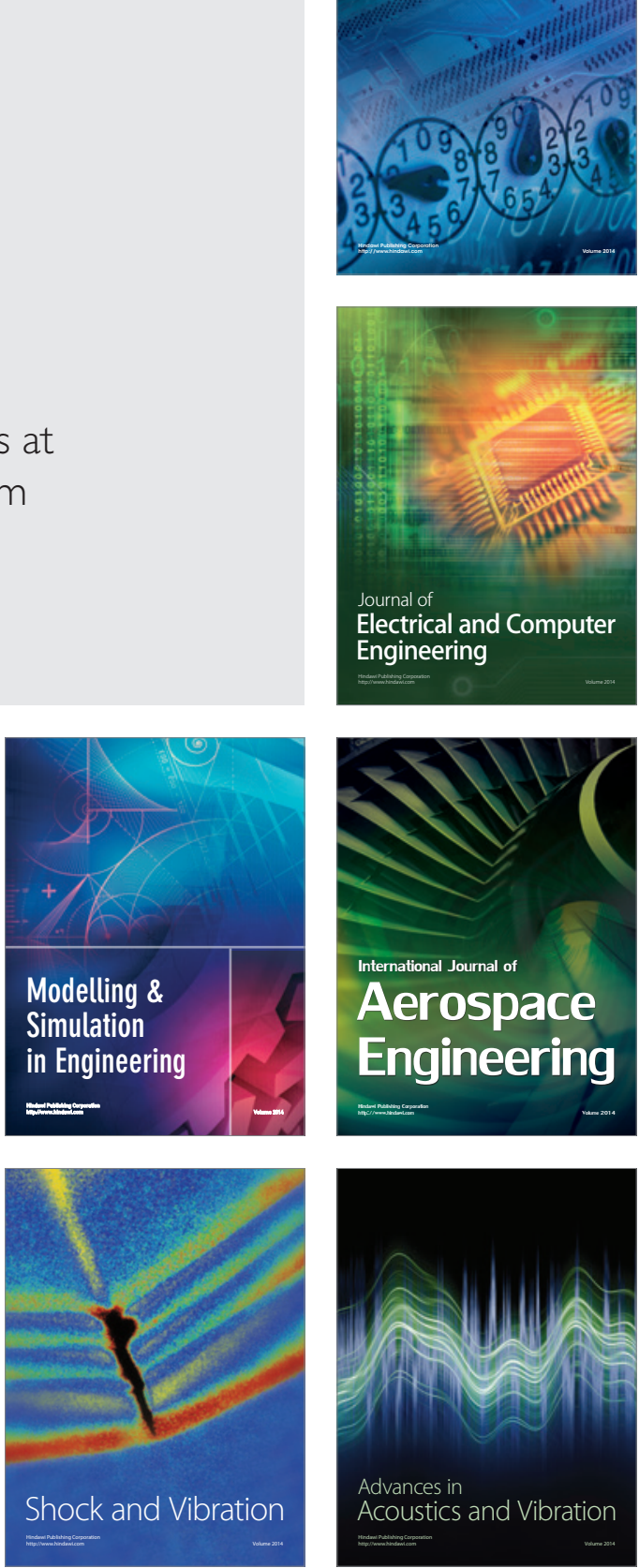